\title{
The Topology of Hyperbolic Attractors on Compact Surfaces
}

Todd L. Fisher

tfisher@byu.edu

Follow this and additional works at: https://scholarsarchive.byu.edu/facpub

Part of the Mathematics Commons

\section{Original Publication Citation}

Ergod. Th. \& Dynam. Sys. (26), 26(5), pp 1511-152.

\section{BYU ScholarsArchive Citation}

Fisher, Todd L., "The Topology of Hyperbolic Attractors on Compact Surfaces" (2003). Faculty Publications. 489.

https://scholarsarchive.byu.edu/facpub/489 
Ergod. Th. \& Dynam. Sys. (2006), XX, 1-5

Printed in the United Kingdom C 2006 Cambridge University Press

\title{
The Topology of Hyperbolic Attractors on
}

\section{Compact Surfaces}

\author{
Todd Fisher \\ University of Maryland, College Park, MD \\ (e-mail: tfisher@math.umd.edu)
}

(Received 27 May 2006)

Abstract. Suppose $M$ is a compact surface and $\Lambda \subset M$ is a nontrivial mixing hyperbolic attractor for some $f \in \operatorname{Diff}(M)$. We show that if $\Lambda$ is a hyperbolic set for some $g \in \operatorname{Diff}(M)$, then $\Lambda$ is a nontrivial mixing hyperbolic attractor or repeller for $g$.

\section{Introduction}

Hyperbolic attractors and repellers are central to many problems in dynamical systems and possess many interesting dynamical properties. The structure of hyperbolic attractors on surfaces has been studied extensively by Plykin [6], Bonatti [1], Williams [9], Zhirov [10] [11] [12], and others. Much of the previous 
work studies the topology of a hyperbolic attractor. In this paper we take a different approach. Namely, we assume some knowledge of the topology of a hyperbolic set $\Lambda$ contained in a surface, and we conclude that $\Lambda$ is a hyperbolic attractor or repeller. Specifically, we prove the following result.

THEOREM 1.1. If $M$ is a compact surface and $\Lambda$ is a nontrivial mixing hyperbolic attractor for a diffeomorphism $f$ of $M$, and $\Lambda$ is hyperbolic for a diffeomorphism $g$ of $M$, then $\Lambda$ is either a nontrivial mixing hyperbolic attractor or a nontrivial mixing hyperbolic repeller for $g$.

Additionally, we are able to show, by counterexample, that the above theorem does not hold if $\operatorname{dim}(M) \geq 3$.

Theorem 1.2. If $\operatorname{dim}(\mathrm{M}) \geq 3$, then there exists a compact set $\Lambda$ hyperbolic for $f, g \in \operatorname{Diff}(M)$ such that $\Lambda$ is a nontrivial mixing attractor for $f$ and neither an attractor nor repeller for $g$.

If the mixing condition in Theorem 1.1 is dropped we have the following weakened result.

THEOREM 1.3. If $M$ is a compact surface and $\Lambda$ is a nontrivial hyperbolic attractor for a diffeomorphism $f$ of $M$, and $\Lambda$ is hyperbolic for a diffeomorphism $g$ of $M$, then there exists an $n \in \mathbb{N}$ and sets $\Lambda_{1}, \ldots, \Lambda_{N}$ where $\Lambda=\bigcup_{i=1}^{N} \Lambda_{i}, \Lambda_{i} \cap \Lambda_{j}=\emptyset$ if $i \neq j$, and each $\Lambda_{i}$ is a mixing hyperbolic attractor or repeller for $g^{n}$.

We note that in $[\mathbf{3}]$ and $[\mathbf{2}]$ topologically one dimensional hyperbolic sets are constructed on compact surfaces that are neither hyperbolic attractors nor repellers. 
There are a number of additional open problems related to Theorems 1.1-1.3.

Problem 1.4. Suppose $\Lambda$ is a locally maximal hyperbolic set for a diffeomorphism $f$ and hyperbolic for a diffeomorphism $g$. Does this imply that $\Lambda$ is locally maximal for $g$ ? or that $\Lambda$ is contained in a locally maximal hyperbolic set for $g$ ?

Problem 1.5. Suppose $M$ is a compact smooth boundaryless manifold of dimension $n$ and $\Lambda$ is a mixing hyperbolic attractor for $f$ with $\operatorname{dim}\left(\mathbb{E}^{u}\right)=n-1$ and hyperbolic for a diffeomorphism $g$. Does this imply that $\Lambda$ is a mixing hyperbolic attractor or repeller for $g$ ?

Related to the first problem we note that in $[\mathbf{3}]$ it is shown that on any manifold, of dimension greater than one, there is an open set of diffeomorphisms containing a hyperbolic set that is not contained in a locally maximal one.

\section{Preliminaries}

We first review some basic facts. A set $X$ is transitive for a continuous map $f$ if there exists a point $x \in X$ such that the closure of the orbit of $x$ is $X$. If $X$ is a compact set of a smooth manifold $M$ and $f$ is a continuous map from $M$ to itself, then $\left.f\right|_{X}$ is transitive if and only if for any open sets $U$ and $V$ of $X$ there exists some $n \in \mathbb{N}$ such that $f^{n}(U) \cap V \neq \emptyset$. A set $X$ is mixing if for any open sets $U$ and $V$ in $X$ there exists an $N \in \mathbb{N}$ such that $f^{n}(U) \cap V \neq \emptyset$ for all $n \geq N$.

From now on we assume that all the maps are diffeomorphisms. A compact set $\Lambda$ invariant under the action of $f$ is hyperbolic if there exists a splitting of the tangent space $T_{\Lambda} f=\mathbb{E}^{u} \oplus \mathbb{E}^{s}$ and positive constants $C$ and $\lambda<1$ such that, for any point 
$x \in \Lambda$ and any $n \in \mathbb{N}$, satisfies:

$$
\begin{aligned}
& \left\|D f_{x}^{n} v\right\| \leq C \lambda^{n}\|v\|, \text { for } v \in E_{x}^{s}, \text { and } \\
& \left\|D f_{x}^{-n} v\right\| \leq C \lambda^{n}\|v\|, \text { for } v \in E_{x}^{u} .
\end{aligned}
$$

The pioneering article by Smale $[8]$ states many of the standard results for hyperbolic sets.

A set $X \subset M$ has an attracting neighborhood if there exists a neighborhood $V$ of $X$ such that $X=\bigcap_{n \in \mathbb{N}} f^{n}(V)$. A set $X \subset M$ has a repelling neighborhood if there exists a neighborhood $U$ of $X$ such that $X=\bigcap_{n \in \mathbb{N}} f^{-n}(U)$. A set $\Lambda \subset M$ is called a hyperbolic attractor (hyperbolic repeller) if $\Lambda$ is a transitive hyperbolic set for a diffeomorphism $f$ with an attracting neighborhood (a repelling neighborhood). A hyperbolic attractor (repeller) is nontrivial if it is not the orbit of a periodic sink (source).

Note, it is always possible to make a smooth change of the metric near the hyperbolic set so that $C=1$. Such a metric is called an adapted metric. For $\epsilon>0$ sufficiently small and $x \in \Lambda$ the local stable and unstable manifolds are respectively:

$$
\begin{aligned}
& W_{\epsilon}^{s}(x, f)=\left\{y \in M \mid \text { for all } n \in \mathbb{N}, d\left(f^{n}(x), f^{n}(y)\right) \leq \epsilon\right\}, \text { and } \\
& W_{\epsilon}^{u}(x, f)=\left\{y \in M \mid \text { for all } n \in \mathbb{N}, d\left(f^{-n}(x), f^{-n}(y)\right) \leq \epsilon\right\} .
\end{aligned}
$$

The stable and unstable manifolds are respectively:

$$
\begin{aligned}
& W^{s}(x, f)=\bigcup_{n \geq 0} f^{-n}\left(W_{\epsilon}^{s}\left(f^{n}(x), f\right)\right), \text { and } \\
& W^{u}(x, f)=\bigcup_{n \geq 0} f^{n}\left(W_{\epsilon}^{u}\left(f^{-n}(x), f\right)\right) .
\end{aligned}
$$

The stable and unstable manifolds of a hyperbolic set are injectively immersed submanifolds.

Prepared using etds.cls 
A hyperbolic set $\Lambda$ is called locally maximal (or isolated) if there exists a neighborhood $V$ of $\Lambda$ in $M$ such that $\Lambda=\bigcap_{n \in \mathbb{Z}} f^{n}(V)$. A hyperbolic set $\Lambda$ possesses a local product structure provided there exist constants $\delta, \epsilon>0$ such that if $x, x^{\prime} \in \Lambda$ and $d\left(x, x^{\prime}\right)<\delta$, then $W_{\epsilon}^{s}(x, f)$ and $W_{\epsilon}^{u}(x, f)$ intersect in exactly one point which is contained in $\Lambda$. For a hyperbolic set locally maximal and possessing a local product structure are equivalent conditions.

We now review properties of Markov partitions. For a hyperbolic set $\Lambda$, the continuity of the stable and unstable distributions implies there exist constants $\epsilon \geq \alpha>0$ such that

$$
f^{-1}\left(W_{\epsilon}^{s}(f(p))\right) \cap f\left(W_{\epsilon}^{u}\left(f^{-1}(q)\right)\right)=W_{\epsilon}^{s}(p) \cap W_{\epsilon}^{u}(q)
$$

is a single point, possibly not contained in $\Lambda$, whenever $p, q \in \Lambda$ satisfy $d(p, q) \leq \alpha$.

A set $R \subset \Lambda$ is a rectangle provided $R$ has diameter less than $\alpha$ and $p, q \in R$ implies that $W_{\epsilon}^{s}(p) \cap W_{\epsilon}^{u}(q) \in R$ where $\epsilon$ and $\alpha$ are as above. A rectangle is proper if $R=\operatorname{cl}(\operatorname{int}(R))$. If $R$ is a rectangle containing a point $x$, then there exists a natural homeomorphism

$$
R \simeq W_{\epsilon}^{s}(x, R) \times W_{\epsilon}^{u}(x, R)
$$

where

$$
W_{\epsilon}^{s}(x, R)=R \cap W_{\epsilon}^{s}(x) \text { and } W_{\epsilon}^{u}(x, R)=R \cap W_{\epsilon}^{u}(x) .
$$

Definition 2.1. A Markov partition of $\Lambda$ is a finite collection of proper rectangles $\left\{R_{i}\right\}_{i=1}^{M}$ covering $\Lambda$ such that

1. if $i \neq j$, then $\operatorname{int}\left(R_{j}\right) \cap \operatorname{int}\left(R_{i}\right)=\emptyset$, 
2. if $z \in \operatorname{int}\left(R_{i}\right)$ and $f(z) \in \operatorname{int}\left(R_{j}\right)$, then $\left.W^{u}\left(f(z), R_{j}\right)\right) \supset f\left(W^{u}\left(z, R_{i}\right)\right)$ and $f\left(W^{s}\left(z, R_{i}\right)\right) \subset W^{s}\left(f(z), R_{j}\right)$.

Next, we review some facts about subshifts of finite type. Let $A=\left[a_{i j}\right]$ be an $n \times n$ matrix with nonnegative integer entries. The graph of $A$ is the directed graph $G_{A}$ with vertices $\mathcal{V}\left(G_{A}\right)=\{1, \ldots, n\}$ and $a_{i j}$ distinct edges with initial state $i$ and terminal state $j$. For the graph $G_{A}$ with edge set $\mathcal{E}$ and adjacency matrix $A$ let $\Sigma$ be the space over $\mathcal{E}$ specified by

$$
\Sigma=\left\{\omega=\left(\omega_{j}\right)_{j \in \mathbb{Z}} \mid t\left(\omega_{j}\right)=i\left(\omega_{j+1}\right) \text { for all } j \in \mathbb{Z}\right\}
$$

where $t\left(\omega_{j}\right)$ is the terminal state of edge $\omega_{j}$ and $i\left(\omega_{j+1}\right)$ is the initial state of edge $\omega_{j+1}$. The map on $\Sigma$ defined by $\sigma(\omega)=\omega^{\prime}$ where $\omega_{j}^{\prime}=\omega_{j+1}$ is called the edge shift map. The subshift of finite type is the space $(\Sigma, \sigma)$. The stable set for a point $t \in \Sigma$ is denoted $W^{s}(t)$ and defined as

$$
W^{s}(t)=\left\{s \in \Sigma \mid \text { there exists } k \in \mathbb{Z} \text { where } s_{i}=t_{i} \text { for all } i \geq k\right\}
$$

The unstable set for a point $t \in \Sigma$ is denoted $W^{u}(t)$ and defined as

$$
W^{u}(t)=\left\{s \in \Sigma \mid \text { there exists } k \in \mathbb{Z} \text { where } s_{i}=t_{i} \text { for all } i \leq k\right\}
$$

A matrix $A$ is irreducible if for each pair $1 \leq i, j \leq n$ there is a $k \in \mathbb{N}$ such that $\left(A^{k}\right)_{i j}>0$. A standard result states that if $A$ is irreducible, then $\Sigma$ contains a transitive point (see [7, p. 277]).

Every adjacency matrix $A$ can be recoded into a block lower triangular matrix, by relabeling the vertices of the graph representing the matrix, where each block is an irreducible component. An irreducible component is a sink (source) if there are 
no outgoing edges from (into) it. There is a dense set of points $S \subset \Sigma_{A}$ such that for all $s \in S$ there exists a $J \in \mathbb{Z}$, a sink $A_{i}$, and a source $A_{j}$ in $A$ depending on $s$ such that for all $k \geq K$ the transition $s_{k}$ to $s_{k+1}$ is contained in $A_{i}$ and $s_{-k-1}$ to $s_{-k}$ is contained in $A_{j}$. (See $[\mathbf{5}$, p. 117] for details.)

Canonically associated to a Markov partition $\mathcal{M}$ is a $\operatorname{SFT}(\Sigma, \sigma)$ with alphabet $\mathcal{A}(\Sigma)=\{1, \ldots, n\}$ such that a transition from $i$ to $j$ is allowed if

$$
f\left(\operatorname{int}\left(R_{i}\right)\right) \cap \operatorname{int}\left(R_{j}\right) \neq \emptyset .
$$

Similarly, a $\operatorname{SFT}(\Sigma, \sigma)$ with factor map $\pi: \Sigma \rightarrow \Lambda$ is said to generate a Markov partition $\mathcal{M}$ if the following hold:

1. the image of a cylinder set $C(i)=\left\{s \in \Sigma \mid s_{0}=i\right\}$ under $\pi$ is a proper rectangle for any $i \in \mathcal{A}(\Sigma)$,

2. a transition from $i$ to $j$ in $\Sigma \operatorname{implies~} f\left(\operatorname{int}\left(R_{i}\right)\right) \cap \operatorname{int} R_{j} \neq \emptyset$, and

3. $\quad$ if $x \in \operatorname{int} R_{i}$ and $f(x) \in \operatorname{int} R_{j}$, then

$$
f\left(W_{\epsilon}^{s}\left(x, R_{i}\right)\right) \subset R_{j} \text { and } f^{-1}\left(W_{\epsilon}^{u}\left(x, R_{j}\right)\right) \subset R_{i} .
$$

For $i \in \mathcal{A}(\Sigma)$ we denote the proper rectangle $R_{i}$ as the image of the cylinder set $C(i)$ under the map $\pi$.

\section{Proofs}

The following theorem will be useful in the the proof of Theorem 1.1.

THEOREM 3.1. [3] If $\Lambda$ is a hyperbolic set and $V$ is a neighborhood of $\Lambda$, then there exists a hyperbolic set $\tilde{\Lambda}$ with a Markov partition such that $\Lambda \subset \tilde{\Lambda} \subset V$.

Prepared using etds.cls 
We now proceed with the proofs.

Proof of Theorem 1.1. The idea of the proof is the following. The proof is divided into two cases. First, if for all $x \in \Lambda$ either

$$
T_{x} W^{u}(x, f)=T_{x} W^{u}(x, g) \text { or } T_{x} W^{u}(x, f)=T_{x} W^{s}(x, g),
$$

then the fact that $W^{u}(x, f)$ is dense in $\Lambda$ for all $x \in \Lambda$ will imply that $\Lambda$ is a mixing hyperbolic attractor or repeller. We do not assume such a choice is consistent for all $x \in \Lambda$. However, we will show this implies that either

$$
\begin{aligned}
& T_{x} W^{u}(x, f)=T_{x} W^{u}(x, g) \text { for all } x \in \Lambda \text { or } \\
& T_{x} W^{u}(x, f)=T_{x} W^{s}(x, g) \text { for all } x \in \Lambda .
\end{aligned}
$$

Second, if

$$
T_{x} W^{u}(x, f) \neq T_{x} W^{u}(x, g) \text { and } T_{x} W^{u}(x, f) \neq T_{x} W^{s}(x, g) \text { for some } x \in \Lambda,
$$

then we will show that any hyperbolic set $\tilde{\Lambda} \supset \Lambda$ with a Markov partition will have nonempty interior and contain a mixing hyperbolic attractor and a mixing hyperbolic repeller for some power of $g$. This will imply that $\Lambda$ contains a mixing hyperbolic attractor $\Lambda_{a}$ and a mixing hyperbolic repeller $\Lambda_{r}$ for some power of $g$. For $x \in \Lambda_{a}$ we will show that $W^{u}(x, f)=W^{u}(x, g)$ so $\Lambda_{a}=\Lambda$. For $y \in \Lambda_{r}$ we will show that $W^{u}(y, f)=W^{s}(y, g)$ so $\Lambda_{r}=\Lambda$, a contradiction.

Before proceeding to the proof we show that the stable and unstable manifolds of $\Lambda$ for $g$ are each one dimensional. Indeed, suppose $\operatorname{dim}\left(E^{s}\right)=2$. Then $W^{u}(x, g)=\{x\}$ for all $x \in \Lambda$. Then for $V$ sufficiently small and $\tilde{\Lambda} \subset V$ a hyperbolic set with a Markov partition for $g$ containing $\Lambda$ we have a $\operatorname{SFT}(\Sigma, \sigma)$ and finite-toone map $\pi$ from $\Sigma$ to $\Lambda$. This implies that the unstable set for any point $s \in \Sigma$ is

Prepared using etds.cls 
finite. Hence, $\Sigma$ consists of a finite number of points, a contradiction. Similarly we can show that $\operatorname{dim}\left(E^{u}\right) \neq 2$.

We now proceed with the proof. Suppose that for all $x \in \Lambda$ either

$$
T_{x} W^{s}(x, g)=T_{x} W^{u}(x, f) \text { or } T_{x} W^{u}(x, g)=T_{x} W^{u}(x, f) .
$$

Fix $x \in \Lambda$ and suppose $T_{x} W^{u}(x, f)=T_{x} W^{u}(x, g)$. Since $W^{u}(x, f)$ is homeomorphic to the reals we know that

$$
T_{y} W^{u}(y, f)=T_{y} W^{u}(y, g) \text { for all } y \in W^{u}(x, f) .
$$

The set $W^{u}(x, f)$ is dense in $\Lambda$, see [7, p. 424]. Hence,

$$
T_{y} W^{u}(y, f)=T_{y} W^{u}(y, g) \text { for all } y \in \Lambda .
$$

A similar argument shows that if $T_{x} W^{u}(x, f)=T_{x} W^{s}(x, g)$, then

$$
T_{y} W^{u}(y, f)=T_{y} W^{s}(y, g) \text { for all } y \in \Lambda \text {. }
$$

Therefore, we know that either

$$
\begin{aligned}
& W^{s}(x, g)=W^{u}(x, f) \text { for all } x \in \Lambda, \text { or } \\
& W^{u}(x, g)=W^{u}(x, f) \text { for all } x \in \Lambda .
\end{aligned}
$$

If $W^{u}(x, g)=W^{u}(x, f)$ for all $x \in \Lambda$, then $\Lambda$ is locally maximal and has an attracting neighborhood for $g$. Indeed, if we extend the adapted metric on $\Lambda$ for $g$ to a neighborhood of $\Lambda$ and fix $\epsilon>0$ sufficiently small, then the set

$$
V=\bigcup_{x \in \Lambda} W_{\epsilon}^{s}(x, g)
$$

is an open neighborhood of $\Lambda$ such that $\bigcap_{n \in \mathbb{N}} g^{n}(V)=\Lambda$. Hence, $\Lambda$ is locally maximal for $g$ with an attracting neighborhood $V$.

Prepared using etds.cls 
If $\Lambda$ is a locally maximal hyperbolic set for $g$ and contains a point $x \in \Lambda$ such that $W^{u}(x) \subset \Lambda$, it follows from Propositions 5 and 6 of $[\mathbf{2}]$ that $\Lambda$ contains a mixing hyperbolic attractor $\Lambda_{a}$ for some power $n$ of $g$. Let $x \in \Lambda_{a}$. The set $W^{u}\left(x, g^{n}\right)$ trivially contains $W^{u}(x, g)$. Since

$$
\Lambda \supset \Lambda_{a} \supset \overline{W^{u}\left(x, g^{n}\right)} \supset \overline{W^{u}(x, g)}=\overline{W^{u}(x, f)}=\Lambda
$$

we know that $\Lambda$ is a nontrivial mixing hyperbolic attractor for $g^{n}$. The set $\Lambda$ is transitive for $g$ since $\Lambda$ is mixing for $g^{n}$. Hence, $\Lambda$ is a nontrivial hyperbolic attractor for $g$. We need to show that $\Lambda$ is mixing for $g$.

The set $\Lambda$ is locally maximal and transitive for $g$, hence $\overline{\operatorname{Per}\left(\left.g\right|_{\Lambda}\right)}=\Lambda$. Fix open sets $U$ and $V$ in $\Lambda$ and $p \in U \cap \operatorname{Per}\left(\left.g\right|_{\Lambda}\right)$. Since $\overline{W^{u}(p, g)}=\Lambda$ we know there exists a $y \in V \cap W^{u}(p, g)$ and $N \in \mathbb{N}$ such that $g^{-n}(y) \in U$ for all $n \geq N$. Hence, $g^{n}(U) \cap V \neq \emptyset$ for all $n \geq N$ and $\Lambda$ is mixing for $g$. If $W^{s}(x, g)=W^{u}(x, f)$ a similar argument shows that $\Lambda$ is a nontrivial mixing hyperbolic repeller for $g$.

Next, suppose for some point $x \in \Lambda$ that

$$
T_{x} W^{s}(x, g) \neq T_{x} W^{u}(x, f) \text { and } T_{x} W^{u}(x, g) \neq T_{x} W^{u}(x, f) .
$$

It then follows that there exists a neighborhood $U$ of $x$ in $\Lambda$ such that for all $y \in U$ we have

$$
T_{y} W^{s}(y, g) \neq T_{y} W^{u}(y, f) \text { and } T_{y} W^{u}(y, g) \neq T_{y} W^{u}(y, f)
$$

Then there exists a constant $\delta_{0}>0$ such that $W_{\delta_{0}}^{u}(x, f) \subset U$.

Let $V$ be a neighborhood of $\Lambda$ and $\tilde{\Lambda} \supset \Lambda$ a hyperbolic set with a Markov partition for $g$ such that $\tilde{\Lambda} \subset V$. Since the rectangles of the Markov partition are 
proper and there are only a finite number of rectangles we know there exists a point $y \in W_{\delta_{0}}^{u}(x, f)$ and $\delta_{1}>0$ such that $W_{\delta_{1}}^{u}(y, f) \subset W_{\delta_{0}}^{u}(x, f) \cap R_{i}$ for some rectangle $R_{i}$ in the Markov partition.

Claim 3.2. $\operatorname{int}(\tilde{\Lambda}) \neq \emptyset$.

Proof. Fix $\epsilon \geq \alpha>0$ such that $\operatorname{diam}\left(R_{i}\right)<\alpha$ for any $R_{i}$ in the Markov partition and $p, q \in R_{i}$ implies $W_{\epsilon}^{s}(p) \cap W_{\epsilon}^{u}(q)$ consists of one point in $R_{i}$. Furthermore,

$$
f^{-1}\left(W_{\epsilon}^{s}\left(f\left(x^{\prime}\right)\right)\right) \cap f\left(W_{\epsilon}^{u}\left(f^{-1}\left(x^{\prime \prime}\right)\right)\right)=W_{\epsilon}^{s}\left(x^{\prime}\right) \cap W_{\epsilon}^{u}\left(x^{\prime \prime}\right)
$$

is a single point, possibly not contained in $\tilde{\Lambda}$, whenever $x^{\prime}, x^{\prime \prime} \in \tilde{\Lambda}$ satisfy $d\left(x^{\prime}, x^{\prime \prime}\right) \leq \alpha$. By the product structure in $R_{i}$ we know that for each $y^{\prime} \in W_{\delta_{1}}^{u}(y, f)$ we have the points

$$
W_{\epsilon}^{s}\left(y^{\prime}, g\right) \cap W_{\epsilon}^{u}(y, g) \text { and } W_{\epsilon}^{s}\left(y^{\prime}, g\right) \cap W_{\epsilon}^{u}(y, g)
$$

are contained in $R_{i}$. Since $W_{\epsilon}^{u}(y, f)$ is diffeomorphic to an open interval this implies that there exist constants $\delta_{u}, \delta_{s}>0$ such that

$$
W_{\delta_{u}}^{u}(y, g) \subset R_{i} \text { and } W_{\delta_{s}}^{s}(y, g) \subset R_{i}
$$

Again using the product structure of $R_{i}$ we have $y \in \operatorname{int}(\tilde{\Lambda})$.

Claim 3.3. The set $\tilde{\Lambda}$ contains a nontrivial hyperbolic attractor $\Lambda_{a}$ and a nontrivial hyperbolic repeller $\Lambda_{r}$.

Proof. Let $(\Sigma, \sigma)$ be the SFT associated with the Markov partition. Then there exists a point $z \in \operatorname{int}(\tilde{\Lambda})$ and $s \in \Sigma$ such that $\pi(s)=z$ and under forward iterates $s$ approaches a sink $A_{i}$ and under backward iterates $s$ approaches a source $A_{j}$. From

Prepared using etds.cls 
Lemma 4 in [2] it follows that for each $x \in \pi\left(A_{i}\right)$ we have $W^{u}(x, g) \subset \pi\left(A_{i}\right)$ and for each $x \in \pi\left(A_{j}\right)$ we have $W^{s}(x, g) \subset \pi\left(A_{j}\right)$. The set $\Lambda_{a}=\pi\left(A_{i}\right)$ is a transitive set. For $\epsilon$ and $\alpha$ as above we know that if $x, y \in \Lambda_{a}$, then $W_{\epsilon}^{s}(x, g) \cap W_{\epsilon}^{u}(y, g)$ consists of one point. Since $W^{u}(y, g) \subset \Lambda_{a} \subset \tilde{\Lambda}$ it follows that $W_{\epsilon}^{s}(x, g) \cap W_{\epsilon}^{u}(y, g) \in \Lambda_{a}$. Hence, $\Lambda_{a}$ has a local product structure and $\Lambda_{a}$ is locally maximal. Let $V$ be an isolating neighborhood for $\Lambda_{a}$. Then there exists an $\epsilon^{\prime \prime}>0$ such that

$$
V^{\prime}=\bigcup_{x \in \Lambda_{a}} W_{\epsilon^{\prime \prime}}^{s}(x, g) \subset V
$$

is an isolating neighborhood of $\Lambda_{a}$. Fix $y \in \bigcap_{n \in \mathbb{N}} g^{n}\left(V^{\prime}\right)$. If $m \in \mathbb{N}$, then $g^{-m}(y) \in \bigcap_{n \in \mathbb{N}} g^{n}\left(V^{\prime}\right)$ since

$$
\bigcap_{n \in \mathbb{N}} g^{m+n}\left(V^{\prime}\right) \supset \bigcap_{n \in \mathbb{N}} g^{n}\left(V^{\prime}\right) .
$$

Hence, $y \in \bigcap_{n \in \mathbb{Z}} g^{n}\left(V^{\prime}\right)=\Lambda_{a}$ and $V^{\prime}$ is an attracting set for $\Lambda_{a}$. Therefore, $\Lambda_{a}$ is a nontrivial hyperbolic attractor for $g$. A similar argument shows that $\Lambda_{r}=\pi\left(A_{j}\right)$ a nontrivial hyperbolic repeller for $g$.

LEMMA 3.4. If

$$
T_{x} W^{s}(x, g) \neq T_{x} W^{u}(x, f) \text { and } T_{x} W^{u}(x, g) \neq T_{x} W^{u}(x, f)
$$

for some $x \in \Lambda$, then the set $\Lambda$ contains a nontrivial hyperbolic attractor and nontrivial hyperbolic repeller for $g$.

Proof. From Claim 3.3 we know that arbitrarily close to $\Lambda$ there exists a nontrivial hyperbolic attractor and nontrivial hyperbolic repeller.

Suppose $\Lambda$ does not contain a hyperbolic attractor for $g$. Then there exists a point $p \in \Lambda$ such that $p$ is a limit point of a sequence $x_{n}$ where each $x_{n}$ is contained 
in a hyperbolic attractor not contained in $\Lambda$. For $\epsilon>0$ sufficiently small the set

$$
\bigcup_{x \in W_{\epsilon}^{u}(p, f)} W_{\epsilon}^{s}(x, g)
$$

is a neighborhood of $p$. Then there exists $x \in W_{\epsilon}^{u}(p, f)$ and some $n \in \mathbb{N}$ such that $x_{n} \in W_{\epsilon}^{s}(x, g)$. Hence, $x$ is contained in a the basin of attraction for a nontrivial hyperbolic attractor $\Lambda_{a}$ for all $n \in \mathbb{N}$. This implies that $g^{n}(x)$ is contained in the basin of attraction for $\Lambda_{a}$. Let $x^{\prime}$ be a limit point for a subsequence of $g^{n}(x)$, then $x^{\prime} \in \Lambda_{a}$ and $x^{\prime} \in \Lambda$ since $\Lambda$ is closed.

Claim 3.5. If $x \in \Lambda$, then

$$
g^{-1}\left(W_{\epsilon}^{u}\left(g^{-n}(x), f\right)\right) \subset W^{u}\left(g^{-n-1}(x), f\right) .
$$

Proof. Note that for all $n \in \mathbb{N}$ we know

$$
g^{-1}\left(W_{\epsilon}^{u}\left(g^{-n}(x), f\right)\right) \subset \Lambda .
$$

Since $\Lambda$ locally is a Cantor set cross an interval this implies that

$$
g^{-1}\left(W_{\epsilon}^{u}\left(g^{-n}(x), f\right)\right) \subset W^{u}\left(g^{-n-1}(x), f\right)
$$

We now return to the proof of the lemma. Suppose that

$$
T_{x^{\prime}} W^{u}\left(x^{\prime}, f\right)=T_{x^{\prime}} W^{u}\left(x^{\prime}, g\right)
$$

Then for $n$ sufficiently large and $\epsilon^{\prime}>0$ sufficiently small we know that $W_{\epsilon^{\prime}}^{u}\left(g^{n}(x), f\right)$ is transverse to $W_{\epsilon^{\prime}}^{s}\left(x^{\prime}, g\right)$. It now follows from the continuity of the unstable distribution and Claim 3.5 that $W^{u}\left(x^{\prime}, f\right)=W^{u}\left(x^{\prime}, g\right)$. Hence, $\Lambda_{a} \subset \Lambda$ since $W^{u}\left(\mathcal{O}_{g}\left(x^{\prime}\right), g\right)$ is dense in $\Lambda_{a}$, a contradiction.

Prepared using etds.cls 
Suppose that

$$
T_{x^{\prime}} W^{u}\left(x^{\prime}, f\right) \neq T_{x^{\prime}} W^{u}\left(x^{\prime}, g\right)
$$

For any periodic point $q_{0} \in \Lambda_{a}$ we know that $W^{u}\left(\mathcal{O}_{g}\left(q_{0}\right), g\right)$ is dense in $\Lambda_{a}$. Since the intersection of $W^{u}\left(x^{\prime}, f\right)$ and $W^{u}\left(x^{\prime}, g\right)$ is transverse this implies there exists a point $q \in \operatorname{Per}\left(\Lambda_{a}\right)$ and $x^{\prime \prime} \in W^{u}\left(x^{\prime}, f\right)$ such that $x^{\prime \prime} \in W^{u}(q, g)$. Then $q \in \Lambda$ since $g^{-n}\left(x^{\prime \prime}\right) \in \Lambda$ for all $n \in \mathbb{N}$.

It now follows from the Lambda Lemma and Claim 3.5 that $W^{u}(q, f)=W^{s}(q, g)$. Since $W^{u}(q, f)$ is dense in $\Lambda$ it now follows that

$$
T_{y} W^{u}(y, f)=T_{y} W^{s}(y, g) \text { for all } y \in \Lambda
$$

a contradiction. Hence $\Lambda$ contains a nontrivial hyperbolic attractor. A similar argument shows that $\Lambda$ contains a nontrivial hyperbolic repeller.

We now return to the proof of the theorem. Let $\Lambda_{a} \subset \Lambda$ be a nontrivial hyperbolic attractor for $g$ and $\Lambda_{r} \subset \Lambda$ be a nontrivial hyperbolic repeller for $g$. From the Spectral Decomposition Theorem for locally maximal hyperbolic sets $[4$, p. 574] there exist sets $\Lambda_{1}, \ldots, \Lambda_{m_{1}}, \Lambda_{1}^{\prime}, \ldots, \Lambda_{m_{2}}^{\prime}$, and constant $n \in \mathbb{N}$ satisfying the following:

- $\Lambda_{a}=\bigcup_{i=1}^{m_{1}} \Lambda_{i}$

- $\Lambda_{i} \cap \Lambda_{j}=\emptyset$ if $i \neq j$

- $g\left(\Lambda_{i}\right)=\Lambda_{\sigma(i)}$ for some permutation $\sigma$

- $g^{n}\left(\Lambda_{i}\right)=\Lambda_{i}$

- $\Lambda_{i}$ is mixing for $g^{n}$,

Prepared using etds.cls 
- $\Lambda_{r}=\bigcup_{i=1}^{m_{2}} \Lambda_{i}^{\prime}$

- $\Lambda_{i}^{\prime} \cap \Lambda_{j}^{\prime}=\emptyset$ if $i \neq j$

- $g\left(\Lambda_{i}^{\prime}\right)=\Lambda_{\sigma^{\prime}(i)}^{\prime}$ for some permutation $\sigma^{\prime}$

- $g^{n}\left(\Lambda_{i}^{\prime}\right)=\Lambda_{i}^{\prime}$, and

- $\Lambda_{j}^{\prime}$ is mixing for $g^{n}$.

For each $\Lambda_{i}$ there exists a neighborhood $U_{i}$ of $\Lambda_{i}$ such that $U_{i} \cap \Lambda_{j}=\emptyset$ if $i \neq j$. Fix $V_{a}$ an attracting neighborhood of $\Lambda_{a}$ for $g$. Then $U_{i} \cap V_{a}$ is an attracting set for $\Lambda_{i}$ under the action of $g^{n}$. Hence, each $\Lambda_{i}$ is a nontrivial mixing hyperbolic attractor for $g^{n}$. Similarly, we can show that each $\Lambda_{j}^{\prime}$ is a nontrivial mixing hyperbolic repeller for $g^{n}$. Let $x \in \Lambda_{a}$, and $y \in \Lambda_{r}$. Then $W^{u}\left(x, g^{n}\right) \subset \Lambda_{i} \subset \Lambda$ for some $1 \leq i \leq m_{1}$. This implies that $W^{u}\left(x, g^{n}\right)=W^{u}(x, f)$ and

$$
\Lambda=\overline{W^{u}(x, f)}=\overline{W^{u}\left(x, g^{n}\right)}=\Lambda_{i}
$$

Similarly, $W^{s}\left(y, g^{n}\right) \subset \Lambda_{j}^{\prime} \subset \Lambda$ for some $1 \leq j \leq m_{2}$ which implies that $W^{s}\left(y, g^{n}\right)=W^{u}(y, f)$ and

$$
\Lambda_{i}=\overline{W^{u}\left(x, g^{n}\right)}=\Lambda=\overline{W^{u}(y, f)}=\overline{W^{s}\left(y, g^{n}\right)}=\Lambda_{j}^{\prime}
$$

a contradiction. Hence, $W^{u}(x, f)=W^{u}(x, g)$ or $W^{u}(x, f)=W^{s}(x, g)$ for all $x \in \Lambda$, and $\Lambda$ is a nontrivial mixing hyperbolic attractor or repeller for $g$.

Proof of Theorem 1.2. Let $f_{0}$ be a diffeomorphism of the open 2-dimensional disk $D^{2}$ such that $f_{0}$ is the identity on a neighborhood of the boundary and $f_{0}$ contains a hyperbolic attractor $\Lambda$. In the appendix of $[\mathbf{3}]$ such a diffeomorphism is constructed.

Prepared using etds.cls 
Let $n \geq 3$ and $f_{1}$ be a diffeomorphism of the open $n-2$ dimensional disk $D^{n-2}$ such that the origin is an attracting fixed point and $f_{1}$ is the identity on a neighborhood of the boundary. Let $f=\left(f_{0}, f_{1}\right)$ and $g=\left(f_{0}, f_{1}^{-1}\right)$.

The set $\Lambda \times\{0\}$ is a hyperbolic attractor for $f$ and hyperbolic for $g$. However, $\Lambda \times\{0\}$ is neither a hyperbolic attractor nor a hyperbolic repeller on $g$.

Let $M$ be a compact manifold with $\operatorname{dim}(M) \geq 3$. Then there exists a sufficiently small open set $U$ in $M$, a local coordinate system $\varphi: U \rightarrow D^{2} \times D^{n-2}$, and diffeomorphisms $F$ and $G$ of $M$ such that $\varphi^{-1} F \varphi=f, \varphi^{-1} G \varphi=g$, and the set $\varphi^{-1}(\Lambda \times\{0\})$ is a hyperbolic for $F$ and $G$, a hyperbolic attractor for $F$, and neither a hyperbolic attractor nor a hyperbolic repeller for $G$.

Proof of Theorem 1.3. Let $\Lambda$ be a hyperbolic attractor for $f$. Then from the Spectral Decomposition Theorem we know that $\Lambda=\Lambda_{1}, \ldots, \Lambda_{N}$ where each $\Lambda_{i}$ is topologically mixing and the $\Lambda_{i}$ are compact disjoint topological sets. Furthermore, there is a power $m \in \mathbb{N}$ of $f$ such that under $f^{m}$ each of the $\Lambda_{i}$ are invariant. Thus each $\Lambda_{i}$ is a mixing hyperbolic attractor under $f^{m}$.

If there is a power $n$ of $g$ such that under $g^{n}$ each of the $\Lambda_{i}$ are hyperbolic sets, then it follows from Theorem 1.1 that each of the $\Lambda_{i}$ are mixing hyperbolic attractors or repellers for $g^{n}$.

To complete the proof of Theorem 1.3 it is sufficient to show the following claim.

Claim 3.6. Given $1 \leq i \leq N$ the set $g\left(\Lambda_{i}\right)=\Lambda_{j}$ for some $1 \leq j \leq N$.

Proof. Let $x \in \Lambda_{i}$. If $g(x) \in \Lambda_{j}$, then there exists a $\delta>0$ such that $g\left(W_{\delta}^{u}(x, f)\right) \subset \Lambda_{j}$. This follows from the continuity of $g$ since each $\Lambda_{i}$ is closed and 
disjoint from any other set $\Lambda_{k}$ where $k \neq i$ and $1 \leq k \leq N$. Suppose $g\left(W^{u}(x, f)\right)$ is not a subset of $\Lambda_{i}$. The set $W^{u}(x, f)$ is a smoothly immersed copy of $\mathbb{R}$, so identifying $W^{u}(x, f)$ with the reals we can find a first point $y \in W^{u}(x, f)$ such that $g(y) \notin \Lambda_{j}$. Let $g(y) \in \Lambda_{k}$ for $k \neq j$. Then there is a $\delta^{\prime}>0$ such that $g\left(W_{\delta^{\prime}}^{u}(y, f)\right) \subset \Lambda_{k}$, a contradiction. Hence, $g\left(W^{u}(x, f)\right) \subset \Lambda_{j}$. Then $g\left(\Lambda_{i}\right) \subset \Lambda_{j}$ since $W^{u}(x, f)$ is dense in $\Lambda_{i}$.

We now show $g\left(\Lambda_{i}\right) \supset \Lambda_{j}$. There exists a sufficiently small neighborhood $U$ of $\Lambda_{i}$ such that $U \cap \Lambda_{k}=\emptyset$ for $k \neq i$. Then $g(U) \cap \Lambda_{j}=g\left(\Lambda_{i}\right) \cap \Lambda_{j}$ since $\Lambda$ is invariant for $g$. Suppose $x \in g\left(\Lambda_{i}\right) \cap \Lambda_{j}$. Then there exists an $\epsilon>0$ such that $W_{\epsilon}^{u}(x, f) \subset g\left(\Lambda_{i}\right) \cap \Lambda_{j}$. As above suppose there is a first point $y \in W^{u}(x, f)$ such that $y$ is not in $g\left(\Lambda_{i}\right)$. Then there exists a $\delta^{\prime}>0$ such that $g\left(W_{\delta^{\prime}}^{u}(y, f)\right)$ is not contained in $\Lambda_{j}$, contradicting the choice of $y$. Hence, $W^{u}(x, f) \subset g\left(\Lambda_{i}\right)$ and $g\left(\Lambda_{i}\right) \supset \Lambda_{j}$ since $W^{u}(x, f)$ is dense in $\Lambda_{j}$. Therefore, $g\left(\Lambda_{i}\right)=\Lambda_{j}$.

\section{REFERENCES}

[1] C. Bonatti and R. Langevin. Difféomorphismes de Smales des surfaces. Asterique, 250, 1998.

[2] T. Fisher. Hyperbolic sets with nonempty interior. Discrete Contin. Dyn. Syst., 15(2):433446, 2006.

[3] T. Fisher. Hyperbolic sets that are not locally maximal. Ergod. Th. Dynamic. Systems, to appear.

[4] B. Hasselblatt and A. Katok. Introduction to the Modern Theory of Dynamical Systems. Cambridge University Press, 1995.

Prepared using etds.cls 
[5] D. Lind and B. Marcus. An Introduction to Symbolic Dynamics and Coding. Cambridge University Press, 1995.

[6] R. V. Plykin. Sources and sinks for a-diffeomorphisms of surfaces. Math. USSR - Sb, 23:233-253, 1974.

[7] C. Robinson. Dynmical Systems Stability, Symbolic Dynamics, and Chaos. CRC Press, 1999.

[8] S. Smale. Differentiable dynamical systems. Bull. Amer. Math. Soc., 73:747-817, 1967.

[9] R. Williams. One-dimensional nonwandering sets. Topology, 6:473-487, 1967.

[10] A. Yu. Zhirov. Hyperbolic attractors of diffeomorphisms of oriented surfaces. Russian Acad. Sci. Sb. Math., 82(1):135-174, 1995.

[11] A. Yu. Zhirov. Hyperbolic attractors of diffeomorphisms of oriented surfaces. ii. enumeration and application to pseudo-anosov diffeomorphisms. Russian Acad. Sci. Sb. Math., 83(1):23-65, 1995.

[12] A. Yu. Zhirov. Hyperbolic attractors of diffeomorphisms of oriented surfaces. iii. a classification algorithm. Sb. Math., 2:221-244, 1995. 